\title{
ACS-PRM: Adaptive Cross Sampling Based Probabilistic Roadmap for Multi-robot Motion Planning
}

\author{
Zhi Yan, Nicolas Jouandeau and Arab Ali Cherif \\ Advanced Computing Laboratory of Saint-Denis (LIASD) \\ Paris 8 University \\ 93526 Saint-Denis, France \\ $\{y z, n, a a\} @ a i . u n i v-p a r i s 8 . f r$
}

\begin{abstract}
In this paper we present a novel approach for multi-robot motion planning by using a probabilistic roadmap (PRM) based on adaptive cross sampling (ACS). The proposed approach, we call ACSPRM, consists of three steps, which are C-space sampling, roadmap building and motion planning. Firstly, an adequate number of points should be generated in C-space on an occupancy grid map by using an adaptive cross sampling method. Secondly, a roadmap should be built while the potential targets and the milestones are extracted by second learning the result of sampling. Finally, the motion of robots should be planned by querying the constructed roadmap. In contrast to previous approaches, our ACS-PRM approach is designed to plan separate kinematic paths for multiple robots to minimize the problem of congestion and collision in an effective way so as to improve the planning efficiency. Our approach has been implemented and evaluated in simulation. The experimental results demonstrate the total planning time can be significantly reduced by our ACS-
\end{abstract} PRM approach compared with previous approaches.

keywords: multi-agent system, motion planning, multi-robot coordination, sampling-based approach

\section{INTRODUCTION}

Motion planning is a fundamental problem in robotics. It could be explained as producing a continuous motion for an agent, that connects a start configuration and a goal configuration, and avoid collision with any static obstacles or other agents in an environment. The agent and obstacle geometry are generally described in a $2 \mathrm{D}$ or $3 \mathrm{D}$ workspace, and the motion could be represented as a path in configuration space. Motion planning algorithms are widely applied in many fields, such as bioinformatics, robotic surgery, industrial automation, planetary exploration and intelligent transportation system.

The multi-agent system (MAS) is proposed to deal with some problems that are difficult or impossible to be solved by a single agent, or to improve the system implementation efficiency in some missions 


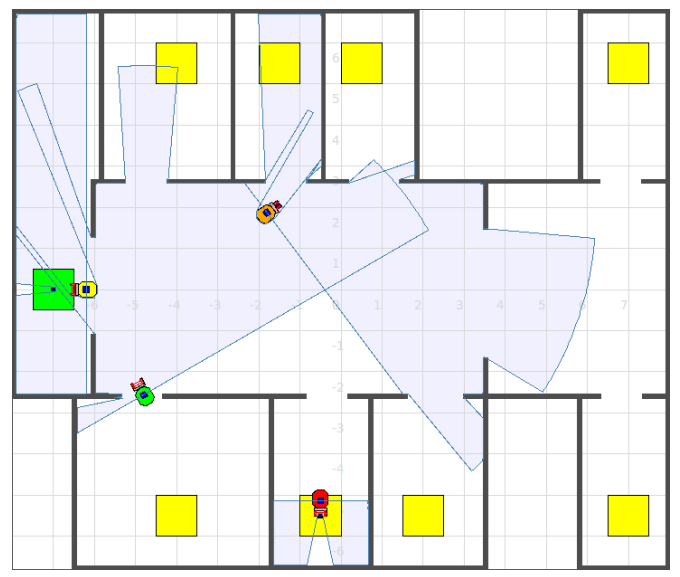

Figure 1: Four robots implement a transportation mission cooperatively. The dark blue piece signifies the goods to be transported. The green area represents the original position of goods, and the yellow area represents the destination, which corresponds to every room, where the goods should be delivered to by the mobile robot.

completed by multi-agent rather than a single agent [1], [2]. The biggest challenge for the MAS is coordination. Without coordination, it will not only lower the system's efficiency, but also lead to the failure of the entire system in extreme cases. Figure 1 shows an example of multi-agent coordination, four robots implement a transportation mission cooperatively, the red robot is delivering a goods, the green robot is on its way back after completing a transportation task, the yellow robot is moving to load a goods, and the orange robot is transporting a goods to the destination location. The coordination of these four robots is obtained by assigning them to different room.

In this paper, we consider the issue of coordinated motion planning for a homogeneous team of autonomous mobile robots in structured environments such as office building, warehouse and container terminal. The larger context of the research is to establish a multi-robot goods transportation system with security, reliability and efficiency. Most of the proposed approaches for multi-robot motion planning usually have the problem of congestion and collision [9], [11]. Because of the transportation issue, a desirable result is that robots need more time to replan their individual local path, thus limit the transportation efficiency, an undesirable result is that the robots are blocked or the goods are lost or damaged and thus fail the transportation mission. We arrange these cases to the waiting situation problem [31]. To handle this practical problem, this paper present a novel approach for multi-robot motion planning by using a probabilistic roadmap planner (PRM) which is based on a manner of adaptive cross sampling (ACS). This approach called ACS-PRM is decomposed into 3 main steps:

- Firstly, we sample an adequate number of points in C-space on an occupancy grid map by using the adaptive cross sampling method.

- Secondly, we build the roadmap by connecting the potential targets and the milestones extracted by second learning the result of sampling. 
- Thirdly, we plan the motion of mobile robots by querying the constructed roadmap based on multi-robot coordination.

The rest of the paper is organized as follows: Section 2 describes an overview of some related works; Section 3 discusses the problem of waiting situation; subsequently, Section 4 describes our ACS-PRM approach; Section 5 presents the experimental results obtained with our approach; and the paper is concluded in Section 6 at last.

\section{RELATED WORK}

Švestka and Overmars [3] presented an approach for multiple nonholonomic car-like robots motion planning in the same static workspace by using probabilistic roadmaps, in which the roadmaps for the composite robot are derived from roadmaps for the underlying simple robots, and the latter is computed by a probabilistic single-robot learning method. The authors introduced the notion of super-graphs for multi-robot path planning, and their implementation covered the construction of the simple roadmap and the super-graphs. This approach is probabilistically complete because a given problem could be solved within a finite amount of time.

Moors et al. [4] presented a graph-based algorithm for coordinate multi-robot motion planning in $2 \mathrm{D}$ indoor environments. The scenario of this research is multi-robot indoor surveillance. The proposed approach takes the limitations and uncertainties of sensors into account, and generates the coordinated motion plan for multiple robots by using A* search algorithm. The authors also introduced a framework based on realistic probabilistic sensor models and worst case assumptions on the intruder's motions in order to compare different approaches and evaluate the coordination performance of the proposed approach.

Clark [5] presented a multi-robot motion planning strategy based on the probabilistic roadmap within a dynamic robot network (DRN) coordination platform. The DRN platform is an ad hoc network, in which a single-query PRM is queried as a centralized planner to plan trajectories for all robots. The PRM planner is optimized to speed queries for multi-robot motion planning by using new sampling strategies. At first, a method of sampling PRM milestones is identified to enable fast coverage of the configuration space. Then, a method of generating PRM milestones is introduced to decrease the planning time. Finally, a endgame region is defined to improve the likelihood of finding solutions when goal configurations are highly constrained.

Saha and Isto [6] presented a strategy for decoupled multi-robot motion planning. The proposed strategy, which aims at improving the reliability of the basic decoupled planning approach, partially merge the two phases of the basic approach. The first phase is to computing a collision-free path to avoid the obstacles in the environment and the other robots, the second phase is coordinating the individual robot motions so that only one robot at a time may enter the area of potential inter-robot interference. The proposed approach searches for motions for a robot and coordination of motions of 
robots along paths already planned while ignoring the robots whose motions have not been planned simultaneously. This approach is inherently incomplete.

Besides, there are some other approaches developed with various policies [7], [8], [9], [10], [11].

In our previous work [31], we considered the issue of using separate topological graphs to coordinated multi-robot motion planning for exploration mission. This work aims at solving the waiting situations in the process of the robot motion planning. In particular, if all the robots take the same topological graph derived from grid map, then they might follow the same exploration path partly or wholly, and this contributes to the problem of waiting situation. We proposed an approach based on sampling environment map iteratively to support the coordinated multi-robot exploration. This research is related to the approach proposed in this paper, even if the methodology is substantially different. Compared to our previous approach, we obtain a significantly reduced mapping time by this approach.

Our recent research focuses on the issue of multi-robot goods transportation. The objective is to complete the transportation mission with high efficiency and low cost. We proposed a heuristic method based on empirical model, which aim at planning the transportation task for each individual robot by estimating the production rate of goods based on multi-robot coordination, so as to improve the system performance. In the module of robot motion planning, we used the wavefront propagation algorithm [27] to global path planning and the nearness diagram algorithm [29] to goal seeking and local obstacle avoidance. Nevertheless, in the experiment, one important reason which influences the system performance is still the waiting situation. Furthermore, if the speed of robot is too fast, then the goods would be damaged or lost in transit because of the collisions with obstacles or others robots. We limited the speed of robot to handle this problem, whereas this strategy limited the efficiency of the whole transportation system as well. Therefore, in this paper we discuss the substance of the problem and propose a novel approach to address such problems in this paper.

\section{WAITING SITUATION PROBLEM}

Multi-agent environments can be cooperative or competitive [12]. To every agent in a team, the other agents can be considered as teammates (cooperative) or movable obstacles (competitive). One of the most important reasons which limit the efficiency of the multi-robot motion planning is the waiting situation such as the congestion and collision between robots. The core of the problem can be considered as the waypoint mutex. Figure 2 depicts a typical waypoint mutex. Two robots move to the same waypoint simultaneously: the red robot move from the top left towards the right and the blue robot move from the right towards the bottom left. Because the waypoint can be assigned only to one robot at a time, then the mutex of the waypoint happens.

Generally, there are two ways to deal with the waypoint mutex as shown in Figure 3. One (Figure $3(\mathrm{a}))$ is to let robots pass the waypoint one by one [3]. The weakness of this strategy is that one robot must wait for another robot to pass. Another way (Figure 3(b)) is to replan the local path in real time for each robot by using some goal seeking obstacle avoidance algorithms such as Vector Field Histogram 


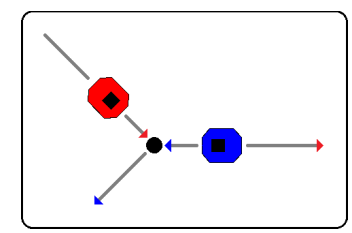

Figure 2: A typical waypoint mutex. The black dot represents the waypoint, the gray segment represents the path, and the red and blue arrow represent the direction of the motion of the corresponding color robots respectively.

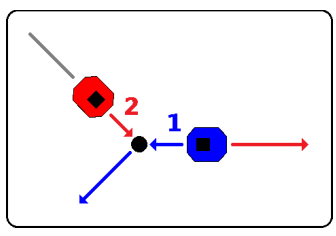

(a)

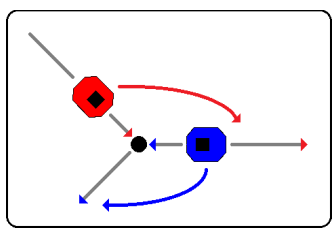

(b)

Figure 3: Two ways to deal with the waypoint mutex. The colored lines represent the motion plan for the corresponding color robots respectively. (a) The two robots pass the waypoint in order, the blue robot pass first and the red robot pass later. (b) The two robots take each other as an obstacle and replan its trajectories in real time.

$(\mathrm{VFH}+)$ [28] or Nearness Diagram (ND) Navigation [29]. The weakness of this strategy is that robots need some time to replan their new trajectory. Consequently, the two ways both extend the time of the motion planning and limit the system efficiency.

If we can plan separate kinematic paths for multiple robots, then the waiting situation caused by waypoint mutex will be significantly reduced. In addition, the problem of multi-robot task allocation (MRTA) [14] should also be considered. Our focus in this paper is on the multi-robot motion planning in structured environments. For instance, an office building could be simply divided into three clusters: corridor, doorway and room. The doorway usually connects the corridor with the room, it is a suitable object for task allocation. Therefore, on the one hand, the problem of waiting situation (especially at the corridors) caused by one path for all robots should be solved. On the other hand, a simple and effective way to coordinate multi-robot motion is to assign different robots to different rooms reachable from the corridors. Besides, more complex environments may require more sophisticated methods such as hand labeled training data [15], [16] or more complex reasoning [17], [18].

Because of the complexity of multi-agent systems (MAS) [7], [13], the target of this paper is not to completely avoid the problem of waiting situation (waypoint mutex), but to minimize the probability of appearance of the waiting situation by using our ACS-PRM approach. Therefore, the coincidence of the waypoint in the plan of two or more robots should be reduced so as to improve the multi-robot motion planning efficiency. 


\section{ACS-PRM: ADAPTIVE CROSS SAMPLING PROBABILIS- TIC ROADMAP}

There are usually two ways to handle the issue of robot motion planning based on the grid representation of the environment in low dimensional space. One is to use the incremental heuristic search algorithm such as $\mathrm{A}^{*}[19]$ or $\mathrm{D}^{*}$ [20]. Another is to use the topological map [21] generated on top of the grid-based map such as Voronoi diagram and straight skeleton. Nevertheless, the number of grids increases rapidly when the size of the environment expends, which make these methods inappropriate for complex and extensive environments. Moreover, these methods are hard to deal with the multi-robot motion planning and always increase the computational load as well.

Sampling-based approaches have been proposed to improve the computational efficiency for robot motion planning. The main idea is to avoid the explicit construction of the obstacle region in the $\mathrm{C}$-space $\left(C_{o b s}\right)$. Unlike the incremental heuristic search and the topological map methods, the sampling-based approaches work well for complex environments and high-dimensional configuration spaces, and they are generally easier to implement. The probabilistic roadmap planner is one of the typical sampling-based approaches. The original PRM technique is introduced by Kavraki et al. [22], which has been shown to perform well in a variety of situations. On the basis of this method, different extensions have been proposed [23], [24], [25], [30]. The approach described in this paper is also an extension of PRM, which is aim at performing multi-robot motion planning efficiently.

To deal with the problem of waiting situation in multi-robot motion planning as mentioned in the last section by using the PRM approach, there are substantially two options:

- In the manner of single-query: when two or more robots need to pass the same waypoint simultaneously, each robot resamples the adjacent region and take the motion of the others into account, then generate a novel local roadmap for local replanning.

- In the manner of multi-query: construct a rich roadmap at the beginning to allow robots to plan a different trajectory from others later.

\subsection{C-space Sampling}

The ACS-PRM approach presented in this paper is a multi-query approach. The first step is C-space sampling, in which an adequate number of points should be generated to represent the free space of the

environment. The main idea of this step is to let a random point $p$ retracts to a position $P(q)$ with the distance $d$ to the obstacle $C_{o b s}$ along horizontal and vertical directions (cross direction).

For autonomous nonholonomic mobile robots, in two dimensions, there are three representational degrees of freedom (DOFs) which are one rotational DOF and two translational DOFs (along or across), but only two controllable DOFs which only move by a forward motion and a steering angle, the configuration space $C$ is the special Euclidean group $S E(2)=\mathbb{R}^{2} \times S O(2)$ where $S O(2)$ is the special orthogonal group of $2 \mathrm{D}$ rotations. To avoid the collision caused by the point retracts too close to the obstacle, we 


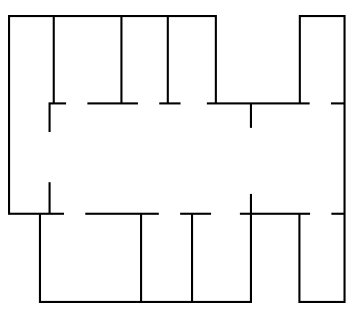

(a)

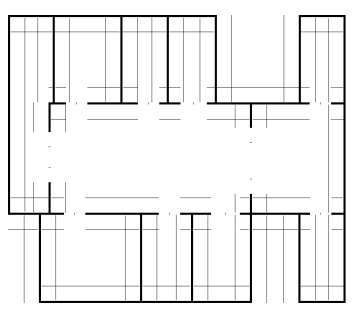

(b)

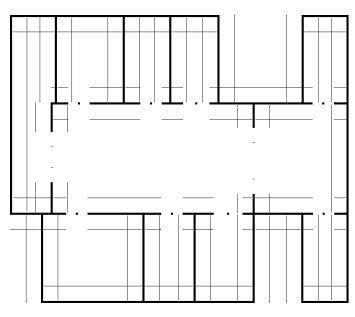

(c)

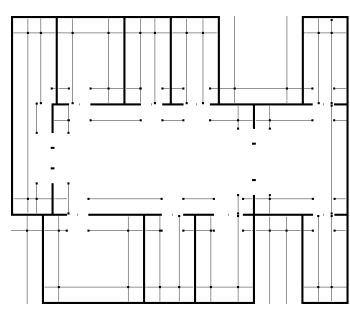

(d)

Figure 4: Generation of the roadmap based on ACS-PRM. (a) The original gridmap, (b) adaptive cross C-space sampling, (c) the potential targets (doorways) extracted, and (d) the milestones extracted.

set the distance $d$ as the sum of the positive number $w$ and the radius $r$ of the minimum circle to cover the robot with centering at the rotation center of robot:

$$
d=r+w,(w>0)
$$

The set of $w$ is to deal with the negative influence of sensor error and it should be adjusted in practical applications.

$C_{o b s}$ represents the set of the obstacle, $\forall q \in C_{o b s}$ define a direction $r_{q}$, then determine a symmetry point $S(q)$ which is an intersection of the open-ray with end $q$ direction $r_{q}$ and another $C_{o b s}$ :

$$
S(q)=\left\{q+t \overrightarrow{r_{q}} \mid t>0\right\} \cap C_{o b s}
$$

where, if $\left\{q+t \overrightarrow{r_{q}} \mid t>0\right\} \cap C_{o b s}=\{q\}$, then define $S(q)=\infty$. Let $\operatorname{dist}(x, y)$ represent the distance between point $x$ and point $y$, then the retraction function can be described as:

$$
P(q)= \begin{cases}q+d \overrightarrow{r_{q}} & \text { if } \operatorname{dist}(q, S(q)) \geq 2 d \\ \frac{q+S(q)}{2} & \text { otherwise }\end{cases}
$$

where $P(q)$ is the position for the point $p$ to retract. In this way, the random points are adapted around to the obstacle (see 4(b)), then:

$$
\left.\operatorname{ACS}-\mathrm{PRM}=\{P(q)\} \mid q \in C_{o b s}\right\}
$$

The implementation of this step is summarized in Algorithm 1. Where the time complexity is $O(n)$ and the space complexity is $O(1)$. This step corresponds the learning phase of classic implementation of PRM.

\subsection{Roadmap Building}

The second step is roadmap building, in which the potential targets and the milestones should be extracted and connected to the roadmap. In the previous step C-space sampling, if there are sufficient 


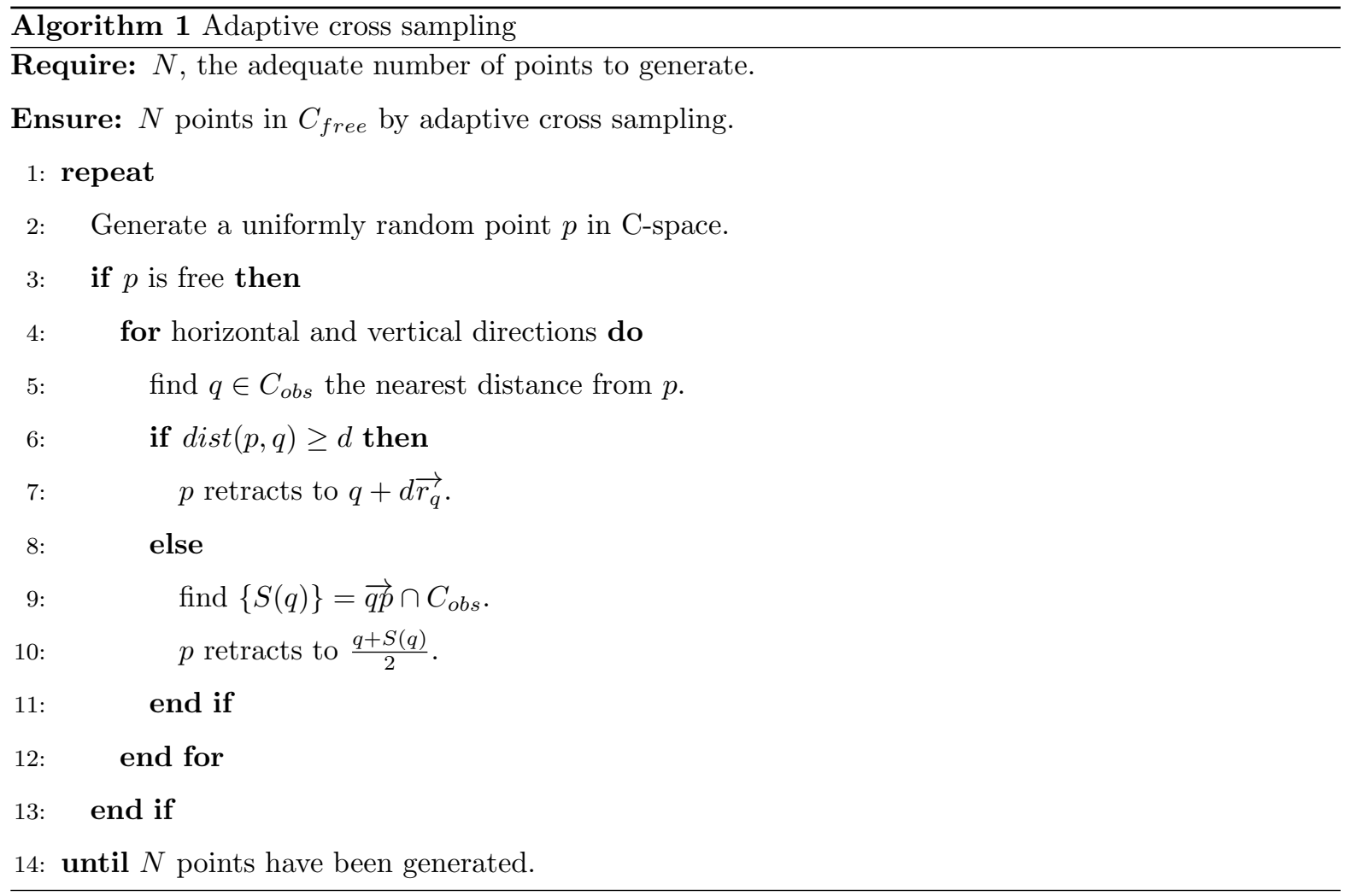

points generated, then the points will gather into segments. The main idea of this step is second learning the graph resulted from the previous step while identifying three types of point as follow:

- In the previous step, if $\operatorname{dist}(p, q)<d$, then $p$ will retract to $\frac{q+S(q)}{2}$ and be labeled as medial axis. Therefore, we find those medial axis segments with length $l$ a small fixed value (in our implementation, we took the thickness of obstacle), and the midpoints of segment are marked as potential target. Figure 4(c) shows the extracted potential targets which are precisely doorways of the structured environment.

- For those segments without containing the potential target, we extract both of the endpoints and mark them as milestone (see Figure 4(d)).

- The points of intersection between two segments are also extracted and marked as milestone (see Figure 4(d)). These milestones have not been used in our experiments, but they will be required for the exploration problem.

This step also corresponds the learning phase of classic implementation of PRM. Figure 4 illustrates the process of generating a roadmap for an example occupancy grid map by using our approach with 200,000 random samples. 


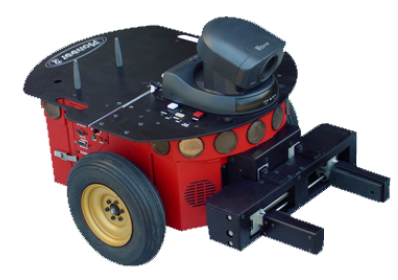

Figure 5: A typical prototype of Pioneer 2-DX robot with gripper

\subsection{Motion Planning}

The third step is motion planning, in which each individual robot's kinematic path should be planned by querying the constructed roadmap. The main idea of this step includes the following three points:

- The potential targets $\left\{t_{i}\right\}$ are considered as the goal nodes for path planning and the objects for task allocation as well. Then the individual $\left\{r_{i}\right\}$ robots are assigned to different potential target: $\left\{r_{i}\right\} \mapsto\left\{t_{i}\right\}$

- To maximize the difference between the paths, we assign the potential target which is the closest from the robot but further from the previous assigned target to the current individual robot: $t=$ further $\left(\operatorname{closest}\left(\left\{t_{i}\right\}, r\right), t_{i-1}\right)$.

- Similar to the classic PRM, we use the fast local planning method (the straight line planner) for the global path planning, except that we choose the path with the minimal number of milestones for the robots invariably.

This step corresponds the query phase of classic implementation of PRM.

\section{EXPERIMENTS}

To evaluate our ACS-PRM approach, we conducted a series of simulation experiments with the wellknown 2D multi-robot simulator Stage [26]. The experiment is to transport a certain amount of goods from one origination to divers destinations by a fleet of mobile robots. The simulated robot is the Pioneer 2-DX robot equipped with a laser range finder providing 361 samples with 180 degrees field of view and a maximum range of 8 meters. Each robot can localize itself based on an abstract localization device which models the implementation of GPS or SLAM. To transport goods, the robots are equipped with a gripper that enable them to sense, pick up and put down the goods, and the carrying capacity is limited to one unit per robot. Figure 5 shows a typical prototype of Pioneer 2-DX robot with gripper.

We used a different number of robots to conduct several experiments in various environments. Two maps (Figure 6) were used in our simulation which are both structured environment. For each map, the green area signifies the original position of goods, and the yellow area signifies the destinations which are always placed in the rooms. For instance, map A has 8 rooms thus 8 destinations, map B has 7 rooms thus 7 destinations. The transportation team size is varied from 2 to 8 robots. On each team size, 


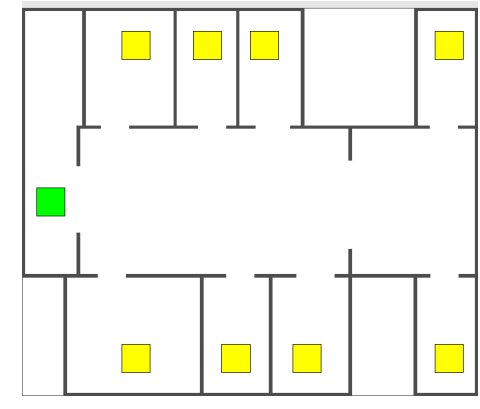

(a) map A

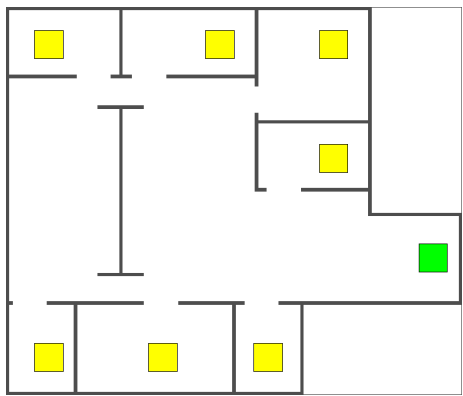

(b) map B

Figure 6: Two environment maps used in our simulation.

10 experimental runs are performed for a transportation mission of 50 goods. The mission objective is to transport the goods to every room equally.

The ratio between real-world time and simulation time is about 1:1. We also compared our approach to the commonly used Voronoi-based approach [21] in which a topological map is built on top of the grid map by using the Voronoi diagram and the critical points are extracted like milestones for mobile robot motion planning. All experiments reported in this paper were carried out on a system with an Intel Core 2 Duo E8400 3.00GHz processor, an Intel Q43 Express chipset and two DDR2 800MHz 1024MB dual channel memory.

In the experiments, we assumed that there exists a central server which is able to communicate with all mobile robots and assign the transportation tasks to each individual robot. The transportation task is to transport the goods from the original position to the destination. We also assumed that all the mobile robots share a common grid map and every one has full information about all others so as to implement path planning and obstacle avoidance in real time coordinately. The ACS-PRM is designed to (but not limited to) plan the kinematic path for nonholonomic mobile robots, and in order to get an objective evaluation of the proposed approach, the drive mode of mobile robot is set to differential-steer, furthermore, the strategy of one pass after the other is applied to deal with the possible waypoint mutex problem.

The results of our experiments are given in Figure 7. We measured the transportation time gained by our approach and compared to the Voronoi-based approach. In each plot, the abscissa denotes the team size of the mobile robots, the ordinate denotes the percentage of the transportation time in the total transportation time, and the error bar indicates the confidence interval of each corresponding gain of robot team size with the 0.95 confidence level. Figure 7 shows that, a transportation time saving of $6.7 \%$ to $12.2 \%$ in map A and $6.1 \%$ to $12.0 \%$ in map B is obtainable under our ACS-PRM approach compared to the Voronoi-based approach. These results proved that our technique could significantly improve the system planning efficiency.

Moreover, we mentioned earlier that our ACS-PRM approach is more effective than our previous approach because the ACS-PRM spends much less time for the learning phase. The experiments shows that, with the new approach, the mapping time are respectively 0.321 seconds and 0.329 seconds for 


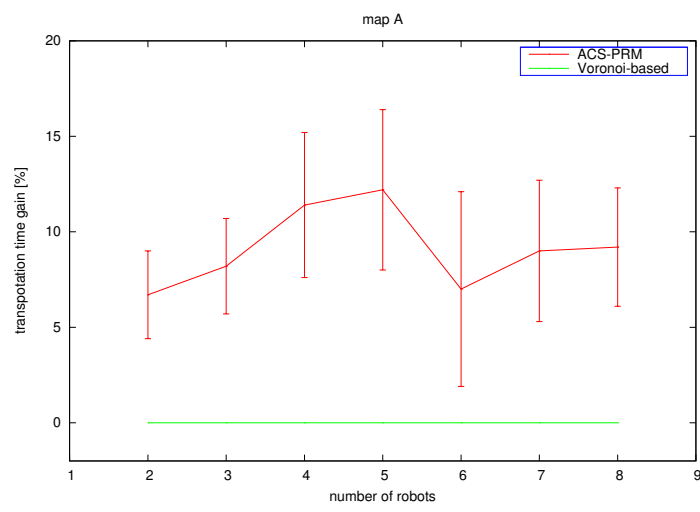

(a) map A

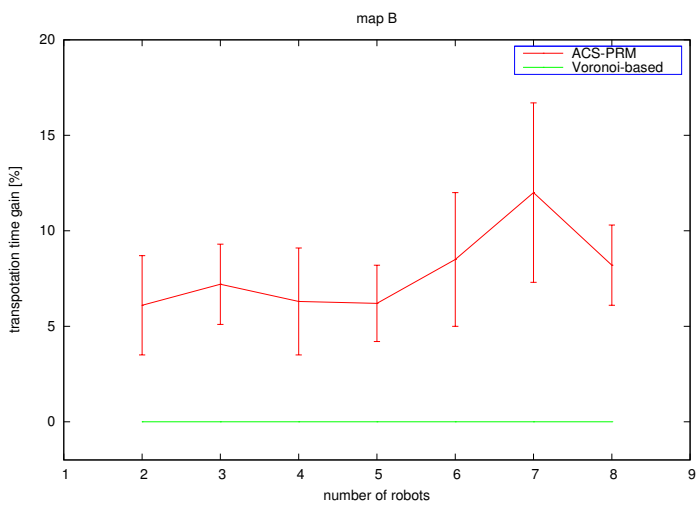

(b) map B

Figure 7: Transportation time gained by using our ACS-PRM approach compared with the Voronoibased approach.

Table 1: Statistics of The Number of Occurrences of The Waypoint Mutex

(a) map A

\begin{tabular}{|c|c|c|c|c|c|c|c|}
\hline \#robots & 2 & 3 & 4 & 5 & 6 & 7 & 8 \\
\hline ACS-PRM & 1.6 & 3.1 & 6.4 & 7.5 & 10.0 & 11.1 & 16.2 \\
\hline Voronoi-based & 15.3 & 18.7 & 26.0 & 26.8 & 19.9 & 23.7 & 27.2 \\
\hline \multicolumn{8}{|c|}{ (b) map B } \\
\hline \#robots & 2 & 3 & 4 & 5 & 6 & 7 & 8 \\
\hline ACS-PRM & 3.8 & 4.3 & 7.1 & 14.9 & 12.8 & 10.3 & 16.7 \\
\hline Voronoi-based & 17.1 & 19.2 & 19.0 & 26.5 & 27.4 & 27.0 & 29.9 \\
\hline
\end{tabular}

map A and map B with 200,000 random samples, which are averages of the 10 runs.

We also counted the average number of the occurrences of waypoint mutex in each map as shown in Table 1. This table shows that the problem of waiting situation is significantly reduced by using our ACS-PRM approach, because our approach is able to plan separate paths for robots, especially in the corridor, whereas we have only one path for all robots with the Voronoi-based approach.

In fact, the technique proposed in this paper also works well with irregular environments (see Figure 8), just need more complex algorithm to extract the milestones.

\section{CONCLUSIONS}

In this paper, we presented a novel approach for coordinated motion planning of multiple robots by using the probabilistic roadmap planner based on a manner of adaptive cross sampling, which we called ACS-PRM. The basic thought of the proposed approach is to build separate kinematic paths for multiple robots to minimize the problem of waiting situation such as collision and congestion caused by waypoint mutex in an effective way, thus to improve the efficiency of automated planning and scheduling. 


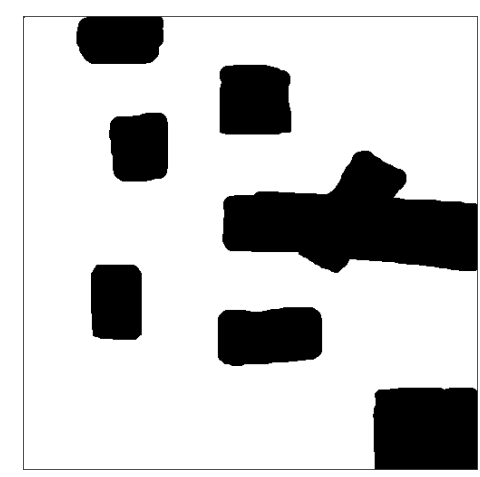

(a)

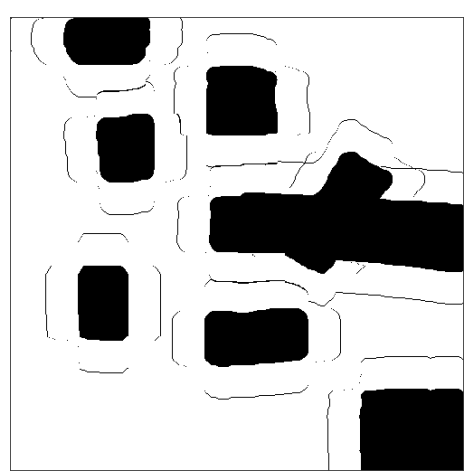

(b)

Figure 8: Irregular environment experiment based on ACS-PRM. (a) The original gridmap, (b) adaptive cross C-space sampling.

The ACS-PRM mainly consists of three steps: C-space sampling, roadmap building and motion planning. In the first step, an adequate number of points are generated to represent the free space of the environment. In the second step, the potential targets and the milestones are extracted and connected to the roadmap by second learning the graph resulted from the previous step. In the third step, the robot's motion planning is done by querying the constructed roadmap. The first two steps correspond the learning phase of classic implementation of PRM, and the last steps corresponds the query phase of classic implementation of PRM.

In consideration of the context about the issue of multi-robot goods transportation, the experiments were conducted to transport a certain amount of goods by a fleet of mobile robots in structured environments. The experimental results demonstrate that, by using our ACS-PRM approach, the total time needed to complete the transportation mission has been significantly reduced compared to the Voronoi-based approach.

In our future work, we will expand our approach to the irregular environments, not just the structured environments. Furthermore, the proposed work in this paper can be also used in some other applications such as exploration mission, automated surveillance, and search and rescue operations. They are our future consideration as well.

\section{REFERENCES}

[1] Y. Cao, A. Fukunaga, and A. Kahng, "Cooperative mobile robotics: Antecedents and directions," Autonomous Robots, vol. 4, no. 1, pp. 7-27, 1997.

[2] G. Dudek, M. Jenkin, E. Milios, and D. Wilkes, "A taxonomy for multi-agent robotics," $A u$ tonomous Robots, vol. 3, no. 4, pp. 375-397, 1996.

[3] P. Švestka and M. H. Overmars, "Coordinated motion planning for multiple car-like robots using probabilistic roadmaps," in Proceedings of the 1995 IEEE International Conference on Robotics 
and Automation (ICRA'95), Nagoya, Japan, May 1995, pp. 1631-1636.

[4] M. Moors, T. Röhling, and D. Schulz, "A probabilistic approach to coordinated multi-robot indoor surveillance," in Proceedings of the 2005 IEEE/RSJ International Conference on Intelligent Robots and Systems (IROS'05), Alberta, Canada, August 2005, pp. 3447-3452.

[5] C. M. Clark, "Probabilistic road map sampling strategies for multi-robot motion planning," in Journal of Robotics and Autonomous Systems, vol. 53, no. 3-4, pp. 244-264, December 2005.

[6] M. Saha and P. Isto, "Multi-robot motion planning by incremental coordination," in Proceedings of the 2006 IEEE/RSJ International Conference on Intelligent Robots and Systems (IROS'06), Beijing, China, October 2006, pp. 5960-5963.

[7] W. Burgard, M. Moors, D. Fox, R. Simmons, and S. Thrun, "Collaborative multi-robot exploration," in Proceedings of the 2000 IEEE International Conference on Robotics and Automation (ICRA'00), San Francisco, CA, USA, April 2000, pp. 476-481.

[8] S. Buck, U. Weber, M. Beetz, and T. Schmitt, "Multi-robot path planning for dynamic environments: a case study," in Proceedings of the 2001 IEEE/RSJ International Conference on Intelligent Robots and Systems (IROS'01), Maui, HI, USA, October 2001, pp. 1245-1250.

[9] A. Solanas and M. A. Garcia, "Coordinated multi-Robot exploration through unsupervised clustering of unknown space," in Proceedings of the 2004 IEEE/RSJ International Conference on Intelligent Robots and Systems (IROS'04), Sendai, Japan, September 2004, pp. 852-858.

[10] R. Regele and P. Levi, "Cooperative multi-robot path planning by heuristic priority adjustment," in Proceedings of the 2006 IEEE/RSJ International Conference on Intelligent Robots and Systems (IROS'06), Beijing, China, October 2006, pp. 5954-5959.

[11] K. M. Wurm, C. Stachniss, and W. Burgard, "Coordinated multi-robot exploration using a segmentation of the environment," in Proceedings of the 2008 IEEE/RSJ International Conference on Intelligent Robots and Systems (IROS'08), Nice, France, September 2008, pp. 1160-1165.

[12] S. Russell and P. Norvig, Artificial Intelligence: A Modern Approach (2nd Edition). Prentice Hall, 2002.

[13] B. P. Gerkey and M. J. Matarić, "Sold!: Auction methods for multirobot coordination," IEEE Transactions on Robotics and Automation, Special Issue on Multi-robot Systems, vol. 18, no. 5, pp. 758-768, 2002.

[14] B. P. Gerkey and M. J. Matarić, "A formal analysis and taxonomy of task allocation in multi-robot systems," The International Journal of Robotics Research, vol. 23, no. 9, pp. 939-954, September 2004. 
[15] E. Brunskill, T. Kollar, and N. Roy, "Topological mapping using spectral clustering and classification," in Proceedings of the 2007 IEEE/RSJ International Conference on Intelligent Robots and Systems (IROS'07), San Diego, CA, USA, October 2007, pp. 3491-3496.

[16] S. Friedman, H. Pasula, and D. Fox "Voronoi Random Fields: Extracting the Topological Structure of Indoor Environments via Place Labeling," in Proceedings of the 20th International Joint Conference on Artificial Intelligence (IJCAI-07), Hyderabad, India, January, 2007, pp. 2109-2114.

[17] P. Beeson, N. K. Jong, and B. Kuipers, "Towards autonomous topological place detection using the extended voronoi graph," in Proceedings of the 2005 IEEE International Conference on Robotics and Automation (ICRA'05), Barcelona, Spain, April 2005, pp. 4373-4379.

[18] Z. Zivkovic, B. Bakker, and B. Kröse, "Hierarchical map building and planning based on graph partitioning," in Proceedings of the 2006 IEEE International Conference on Robotics and Automation (ICRA'06), Orlando, FL, USA, May 2006, pp. 803-809.

[19] P. E. Hart, N. J. Nilsson, and B. Raphael, "A formal basis for the heuristic determination of minimum cost paths," IEEE Transactions on Systems Science and Cybernetics, vol. 4, no. 2, pp. 100-107, July 1968.

[20] A. Stentz, "Optimal and efficient path planning for partially-known environments," in Proceedings of the 1994 IEEE International Conference on Robotics and Automation (ICRA'94), San Diego, CA, USA, May 1994, pp. 3310-3317.

[21] S. Thrun, "Learning metric-topological maps for indoor mobile robot navigation," Artificial Intelligence, vol. 99, no. 1, pp. 21-71, February 1998.

[22] L. E. Kavraki, P. Švestka, J.-C. Latombe, and M. H. Overmars, "Probabilistic roadmaps for path planning in high-dimensional configuration spaces," IEEE Transactions on Robotics and Automation, vol. 12, no. 4, pp. 566-580, 1996.

[23] N. M. Amato, O. B. Bayazit, L. K. Dale, C. Jones, and D. Vallejo, "OBPRM: An obstacle-based PRM for 3D workspaces," in Proceedings of the Workshop on Algorithmic Foundations of Robotics (WAFR'98), Houston, TX, USA, March 1998, pp. 155-168.

[24] S. A. Wilmarth, N. M. Amato, and P. F. Stiller, "MAPRM: A probabilistic roadmap planner with sampling on the medial axis of the free space," in Proceedings of the 1999 IEEE International Conference on Robotics and Automation (ICRA'99), Detroit, MI, USA, May 1999, pp. 1024-1031.

[25] G. Song and N. M. Amato, "Randomized motion planning for car-like robots with C-PRM," in Proceedings of the 2001 IEEE/RSJ International Conference on Intelligent Robots and Systems (IROS'01), Maui, HI, USA, October 2001, pp. 37-42. 
[26] B. P. Gerkey, R. T. Vaughan, and A. Howard, "The player/stage project: Tools for multi-robot and distributed sensor systems," in Proceedings of the 11th International Conference on Advanced Robotics (ICAR'03), Coimbra, Portugal, June 2003, pp. 317-323.

[27] J.-C. Latombe, Robot Motion Planning. Kluwer Academic Publishers, 1991.

[28] I. Ulrich and J. Borenstein, "VFH+: Reliable obstacle avoidance for fast mobile robots," in Proceedings of the 1998 IEEE International Conference on Robotics and Automation (ICRA'98), Leuven, Belgium, May 1998, pp. 1572-1577.

[29] J. Minguez and L. Montano, "Nearness diagram (ND) navigation: Collision avoidance in troublesome scenarios," IEEE Transactions on Robotics and Automation, vol. 20, no. 1, pp. 45-49, February 2004.

[30] N. Jouandeau, "Algorithmique de la planification de mouvement probabiliste pour un robot mobile," Ph.D. dissertation, Paris 8 University, December 2004.

[31] Z. Yan, N. Jouandeau, and A. Ali Cherif. "Sampling-based multi-robot exploration," in Proceedings of the Joint 41th International Symposium on Robotics and 6th German Conference on Robotics (ISR/ROBOTIK 2010), Munich, Germany, June 2010, pp. 44-49. 